\title{
THE UNIFORM BOUNDEDNESS PRINCIPLE FOR ORDER BOUNDED OPERATORS
}

\section{CHARLES SWARTZ}

\author{
Department of Mathematical Sciences \\ New Mexico State University \\ Las Cruces, NM 88003 \\ (Received April 18, 1988)
}

\begin{abstract}
Under appropriate hypotheses on the spaces, it is shown that a sequence of order bounded linear operators which is pointwise order bounded is uniformly order bounded on order bounded subsets. This result is used to establish a
\end{abstract} Banach-Steinhaus Theorem for order bounded operators.

KEY WORDS AND PHRASES: lattice, order bounded operator, uniform boundedness principle, Banach-Steinhaus Theorem.

1980 MATHEMATICS SUBJECT CLASSIFICATION: $46 A 40$.

\section{INTRODUCTION}

In this note we consider the problem of obtaining a version of the classical Uniform Boundedness Principle of functional analysis for linear operators between vector lattices. If $X$ is a Banach space and $Y$ is a normed linear space, the Uniform Boundedness Principle then asserts that any sequence $\left\{\mathrm{T}_{1}\right\}$ of continuous linear operators from $X$ into $Y$ which is pointwise bounded on $X$ is such that the sequence of operator norms $\left\{11 T_{1} \|\right\}$ is bounded ([1] 4). It is easy to see that the condition that the $\left\{\left\|T_{i}\right\|\right\}$ are bounded is equivalent to the condition that the sequence $\left\{T_{i}\right\}$ is uniformly bounded on bounded subsets of $X$ (see the discussion in [1] 4). Thus, a possib]e version of the Uniform Boundedness Principle for order bounded linear operators $\left\{T_{1}\right\}$ between vector lattices $X$ and $Y$ might be that if $\left\{T_{1}\right\}$ is pointwise order bounded on $X$, then $\left\{T_{1}\right\}$ is uniformly order bounded on order bounded subsets of $X$. We will show below in Example 1 that such a straightforward analogue of the Uniform Boundedness Principle does not hold even if both $X$ and $Y$ are Banach lattices. However, by imposing additional conditions on 
the spaces and by employing the matrix methods of [1], we will obtain an order version of the Uniform Boundedness Principle in Theorem 3 below. Using the Uniform Boundedness Principle, we also establish a version of the Banach-Steinhaus Theorem for order bounded operators which generalizes a result of Nakano.

2. RESULTS

First, consider the following example which shows that a straightforward analogue of the Uniform Boundedness Principle does not hold for order bounded linear operators between Banach lattices. (A linear operator $T$ between vector lattices or Riesz spaces $X$ and $Y$ is order bounded if $T$ carries order bounded subsets of $X$ into order bounded subsets of $Y$, where a subset $A$ of a vector lattice $X$ is order bounded if there is an order interval $[-u, u]=\{v \in X:-u \leq v \leq u\}$ such that $A \subseteq[-u, u]$ (in general, we conform to the notation and terminology of [5]).)

EXAMPLE 1. Let $X=L^{1}[0,1]$ and $Y=c_{0}$ and assume that these spaces have the usual pointwise ordering. For $f \in L^{1}[0,1]$, set $f_{k}=\int_{0}^{1} f(t) s i n \pi k t d t$. Define $T_{k}: X \rightarrow Y$ by $T_{k} f=\sum_{j=1}^{k} f_{j} e_{j}$, where $e_{j}$ is the element of $c_{0}$ with a 1 in the $j^{\text {th }}$ coordinate and 0 in the other coordinates. Each $T_{k}$ is order bounded since if $|f| \leq g$ in $x$, then $\left|f_{j}\right| \leq \int_{0}^{1} g=a$ and $\left|T_{k} f\right| \leq \sum_{j=1}^{k} a e_{j}$. Also, the sequence $\left\{T_{k}\right\}$ is pointwise order bounded on $x$ since if $f \in X$, then $\left|T_{k} f\right| \leq \sum_{j=1}^{\infty}\left|f_{j}\right| e_{j} \in c_{0} \quad$ (note $\quad\left\{\left|f_{j}\right|\right\} \in c_{0} \quad$ by the Riemann-Lebesgue Lemma). However, $\left\{T_{k}\right\}$ is not uniformly order bounded on order bounded subsets of $x$ since if $\boldsymbol{P}_{k}(t)=\sin \pi k t$, then $\quad\left\{\boldsymbol{P}_{k}\right\}$ is order bounded in $x \quad\left(\left|\boldsymbol{P}_{k}\right| \leq 1\right)$ but $\left\{T_{i}\left(P_{i}\right)\right\}=\left\{e_{i} / 2\right\}$ is not order bounded in $c_{0}$.

Note that both $X$ and $Y$ in this example are Banach lattices and both are Dedekind complete.

In order to obtain our version of the Uniform Boundedness Principle for ordered spaces, we first obtain a matrix theorem which is the analogue for ordered spaces of the matrix result given in [1] 2.1 .

Throughout the remainder of this note we let $X$ and $Y$ denote Riesz spaces (vector lattices). A sequence $\left\{x_{k}\right\}$ in $x$ is $u$-convergent to $x$, where $u \geq 0$, if there exists a scalar sequence $t_{k} \downarrow_{0}$ such that $\left|x_{k}-x\right| \leq t_{k} u$; we write $u-\lim x_{k}=x$. The element $u$ is called a convergence regulator for $\left\{x_{k}\right\}$. The sequence $\left\{x_{k}\right\}$ is relatively uniformlv convergent to $x$ if $\left\{x_{k}\right\}$ is u-convergent to $x$ for some convergence regulator $u \geq 0$; we write $r-1 \mathrm{~m} x_{k}=x$. 
LEMMA 2. ([4] 2.1) Let the infinite matrix $\left[x_{1 j}\right], x_{i j} \in X$, be such that its rows and columns are $u$-convergent to 0 . Let $\epsilon_{i f}>0, \epsilon_{i f} \in R$. Then there exists an increasing sequence of positive integers $\left\{p_{1}\right\}$ such that $\left|x_{p_{1} p_{j}}\right| \leq \epsilon_{i j} u$ for $1 \neq j$.

PROOF. Put $p_{1}=1$. Since $\left\{x_{1 j}\right\}$ and $\left\{x_{11}\right\}$ are u-convergent to 0 , there exists $p_{2}>p_{1}$ such that $\left|x_{1 p_{2}}\right| \leq \epsilon_{12} u$ and $\left|x_{p_{2} 1}\right| \leq \epsilon_{21} u$. Similarly, there is $\quad p_{3}>p_{2}$ such that $\left|x_{p_{1} p_{3}}\right| \leq \epsilon_{13} u,\left|x_{p_{2} p_{3}}\right| \leq \epsilon_{23} u,\left|x_{p_{3} p_{2}}\right| \leq \epsilon_{32} u$ and $\left|x_{p_{3} p_{1}}\right| \leq \epsilon_{31} u . \quad$ Now continue.

We now prove our Uniform Boundedness Principle for order bounded operators. Recall that if $Y$ is Dedekind complete, then a linear map $T: X \rightarrow Y$ is order bounded if and only if $T$ is regular ([5] VIII.2.2).

THEOREM 3. Let $X$ be Dedekind $\sigma$-complete and let $Y$ be Dedekind complete and have an order unit $u$. If $T_{1}: X \rightarrow Y$ is a sequence of order bounded linear maps which 18 pointwise order bounded on $X$, then $\left\{T_{1}\right\}$ is uniformly order bounded on order bounded subsets of $X$.

PROOF. If the conclusion fails, there 18 an interval [-w, w] in $x$ such that $\left\{T_{1}([-w, w]): i \in N\right\}$ is not order bounded. Thus, for each 1 there exist $x_{1} \in[-w, w]$ and $m_{i}$ such that $T_{m_{1}} x_{1} \in 1^{4}[-u, u]$. For notational convenience, assume that $n_{1}=1$. Then

$$
T_{1} x_{1} \in 1^{4}[-u, u]
$$

Now consider the matrix $M=\left[(1 / 1) T_{1}\left(x_{j} / j^{2}\right)\right]$. For each $j,\left\{T_{1}\left(x_{j} / j^{2}\right)\right\}$ is order bounded so the $j^{\text {th }}$ column of $M$ is relatively uniformly convergent to 0 . The sequence $\left\{x_{j} / j^{2}\right\}$ is relatively uniformly convergent to 0 , and since each $T_{1}$ is sequentially continuous with respect to relative uniform convergence ([5] VIII 1.2), the $i^{\text {th }}$ row of $M$ is also relatively uniformly convergent to 0 . Thus, the rows and columns of $M$ are $u$-convergent to 0 . By Lema 2 there is an increasing sequence of positive integers $\left\{p_{i}\right\}$ such that $\left|\left(1 / p_{1}\right) T_{p_{i}}\left(x_{p_{j}} / p_{j}{ }^{2}\right)\right| \leq 2^{-i-J_{v}}$ for $1 \neq j$. Again for notational convenience, we assume that $p_{1}=1$. Since $x$ is o-complete and $\left|x_{j}\right| \leq w$, the series $\sum x_{j} / j^{2}$ is absolutely order convergent to an element $x \in X \quad$ ([5] IV. 9); moreover, this series is actually $w$-convergent in $X$ since $\quad\left|x-\sum_{j=1}^{n} x_{j} / j^{2}\right| \leq \sum_{j=n+1}^{\infty}\left|x_{j}\right| / j^{2} \leq\left(\sum_{j=n+1}^{\infty} 1 / j^{2}\right) w$. From the continuity of $T_{i}$ with respect to relative uniform convergence, we have 


$$
\begin{aligned}
& \left|(1 / i) T_{i}\left(x_{i} / i^{2}\right)\right| \leq\left|\sum_{j=1}^{\infty}(1 / i) T_{i}\left(x_{j} / j^{2}\right)\right|+\left|\sum_{\substack{j=1 \\
j \neq i}}^{\infty}(1 / i) T_{i}\left(x_{j} / j^{2}\right)\right| \\
& \leq\left|(1 / 1) T_{i}(x)\right|+\sum_{\substack{j=1 \\
j \neq i}}^{\infty}\left|(1 / i) T_{i}\left(x_{j} / j^{2}\right)\right| \\
& \leq\left|(1 / 1) T_{1}(x)\right|+\sum_{\substack{j=1 \\
j \neq 1}}^{\infty} 2^{-1-j} \\
& \leq(1 / i)\left|T_{i}(x)\right|+2^{-i} v
\end{aligned}
$$

where we have used the order completeness of $Y$ to insure that the series on the right hand side of (2) are convergent. Both terms on the right hand side of (2) are order bounded. Hence, there exists $k$ such that $(1 / 1)\left|T_{i}\left(x_{i} / 1^{2}\right)\right| \in k[-u, u]$ for all 1 . Putting $i=k$ contradicts (1) and the result is established.

Note that the range space $c_{0}$ in Example 1 is Dedekind complete, but does not have an order unit.

It is perhaps worthwhile noting that if $\left\{\mathrm{T}_{1}\right\}$ is uniformly order bounded on order bounded subsets of $X$, then the sequence of moduli $\left\{\left|T_{1}\right|\right\}$ also has this property. For if $\quad 0 \leq x \leq u \quad$ and $\quad\left|T_{i}[0, u]\right| w$, then $\left|\mathrm{T}_{1}\right| \mathrm{x}=\sup \left\{\left|\mathrm{T}_{1} \mathrm{z}\right|: 0 \leq \mathrm{z} \leq \mathrm{x}\right\} \leq \mathrm{w}\left([5] \operatorname{VIII.2)}\right.$ so $\left|\mathrm{T}_{1}\right|[0, u] \subseteq[0, w]$.

From Theorem 3 we can also obtain an order analogue of the equicontinuity conclusion of the classical Uniform Boundedness Principle. Recall that if $\left\{\mathrm{T}_{1}\right\}$ is a sequence of continuous linear operators from a normed space $x$ into a normed space $Y$, then $\left\{T_{i}\right\}$ is equicontinuous if and only if $\left\{T_{i}\right\}$ is uniformly bounded on bounded subsets of $x$ if and only if $T_{1} x_{1} \rightarrow 0$ whenever $x_{1} \rightarrow 0$ if and only if $\underset{j}{\lim } \mathrm{T}_{i} \mathrm{x}_{j}=0$ uniformly in 1 whenever $x_{j} \rightarrow 0$ ([1] 4.5). It is easy to establish order analogues of these equicontinuity conditions for operators which satisfy the conclusion of Theorem 3 . We give order analogues of these conditions below and consider the relationship between them. If for each a $\in A,\left\{x_{1 a}\right\}$ is a sequence in $x$, we say that $\left\{x_{1 a}\right\}$ is $u$-convergent to $e$ uniformly for $a \in A$ if there exists a sequence $t_{1} \downarrow_{0}$ such that $\left|x_{1 a}\right| \leq t_{1} u$ for all a $\in A$; we write $u-\underset{1}{11 m} x_{1 a}=0$ uniformly for $a \in A$. 
PROPOSITION 4. Let $\mathrm{T}_{\mathrm{i}}: \mathrm{X} \rightarrow \mathrm{Y}$ be order bounded. Consider

(i) $\left\{\mathrm{T}_{1}\right\}$ is uniformly order bounded on order bounded subsets of $x$.

(11) if $r-\lim x_{j}=0$, then $v-\underset{j}{\lim T_{1} x_{j}=0}$ uniformly for $1 \in N$ for some $v \in Y, v \geq 0$,

(1ii) $r-1$ im $T_{1} x_{i}=0$ whenever $r-\lim x_{i}=0$.

Always (1) implies (1i) implies (1ii). If $Y$ is Archimedean and has the boundedness property ([3] 1.5.12), then (i11) implies (1).

PROOF. Assume (i) holds. To establish (ii), there exists $t_{j} f^{\infty}$ such that $\left\{t_{j} x_{j}\right\}$ is relatively uniformly convergent to 0 ([4] VI.4). Since $\left\{t_{j} x_{j}\right\}$ is order bounded, by (i) there is $v \in Y$ such that $\left|T_{i}\left(t_{j} x_{j}\right)\right| \leq v$ for all $1, j$. Then $\left|T_{i} x_{j}\right| \leq\left(1 / t_{j}\right) v$ implies (1i).

Clearly (ii) implies (iii). Assume (iii) and that $Y$ is Archimedean with the boundedness property. Let $u \geq 0, u \in X$. By the boundedness property, it suffices to show that if $\left\{T_{k_{1}}\right\}$ is a subsequence, $\left|x_{1}\right| \leq u$ and $t_{1} \downarrow 0$, then $r-\lim t_{i} T_{k_{1}} x_{1}=0$. But, since $u-l i n t_{i} x_{1}=0$, this follows from (111).

Theorem 3 gives sufficient conditions for the equicontinuity condition ( 1 ) and, therefore, (11) and (11i), to hold.

As in the classical case we can apply the Uniform Boundedness Principle given in Theorem 3 to obtain a Banach-Steinhaus type result for order bounded operators ([1] 5.1). There is an order version of the Banach-Steinhaus Theorem for linear functionals due to Nakano given in [5] IX. 1.1, and a general form of the Banach-Steinhaus Theorem for operators between vector lattices given in [4]. The results in [4] treat a different class of operators than that considered below in Corollary 5 and the assumptions on the spaces are not as restrictive.

COROLLARY 5. Let $X, Y$ be as in Theorem 3 and let $T_{1}: X \rightarrow Y$ be order bounded. If $0-1$ in $T_{1} X=T x$ exists for each $X \in X$, then $T: X \rightarrow Y$ is an order bounded linear operator.

PROOF. For each $x,\left\{T_{1} x\right\}$ is order bounded since the sequence 18 order convergent. Therefore, from Theorem $3,\left\{T_{1}\right\}$ is uniformly order bounded on order bounded subsets of $x$. If $[u, v]$ is an order interval in $x$, then there is an order interval $[-w, w]$ in $Y$ such that $T_{1}([u, v]) \subseteq[-w, w]$ for all i. Hence. $T([u, v]) \subseteq[-w, w]$ and $T$ is order bounded.

The sequence of operators $\left\{T_{1}\right\}$ in Example 1 is pointwise order convergent to the operator $T: X \rightarrow Y$ given by $T f=\sum_{j=1}^{\infty} f_{j} e_{j}$ However, $T$ is not order bounded since $\left\{\boldsymbol{T P}_{j}\right\}=\left\{\mathrm{e}_{\mathrm{j}} / 2\right\}$, where $\boldsymbol{\varphi}_{j}(t)=\sin \pi_{\mathrm{j} t}$. This shows that in general the 
Banach-Steinhaus result will not hold if the range space is merely Dedekind complete.

The author would like to thank Joe Kist for his kind assistance.

\section{REFERENCES}

1. P. Antosik and C. Swartz, Matrix Methods in Analysis, Lecture Notes in Mathematics 1113, Springer-Verlag, 1985.

2. W.A.J. Luxemburg and A.C. Zaanen, Riesz Spaces I, North Holland, 1971.

3. A.L. Peressini, Ordered Topologlcal Vector Spaces, Harper and Row, 1967.

4. C. Swartz, The Banach-Steinhaus Theorem for ordered spaces, Proceedings of the Conference GRAC, Dubrovnik, Yugoslavia, 1987, to appear.

5. B.z. Vulikh, Introduction to the Theorv of Partially ordered spaces, Wolters-Noordhoff, 1967. 


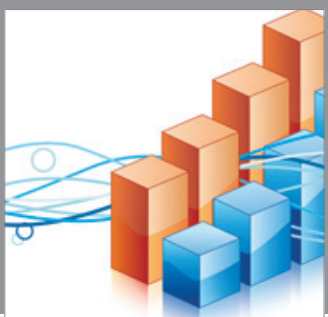

Advances in

Operations Research

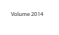

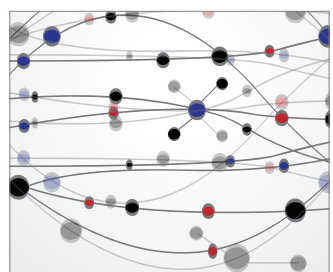

\section{The Scientific} World Journal
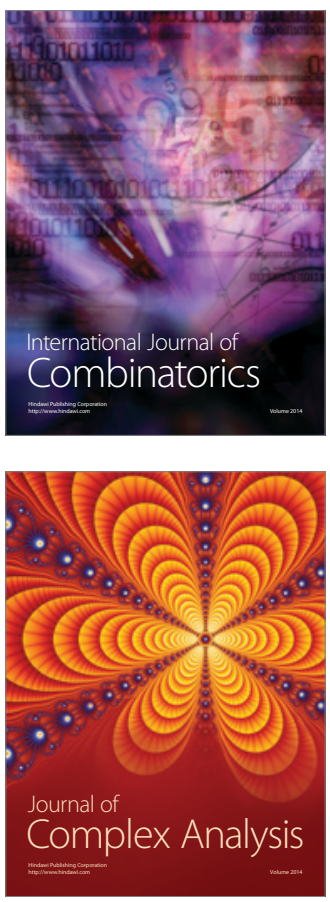

International Journal of

Mathematics and

Mathematical

Sciences
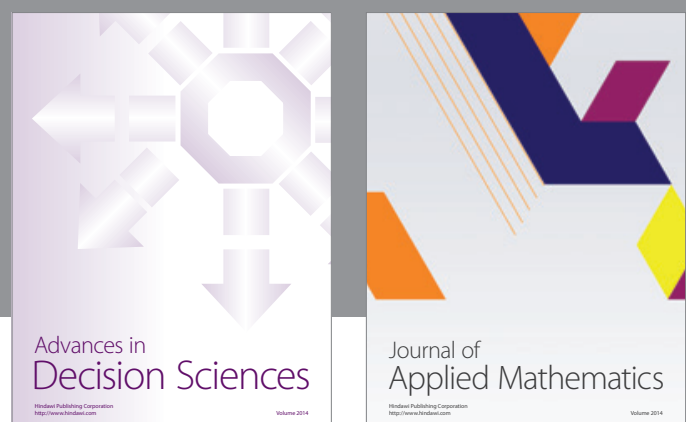

Journal of

Applied Mathematics
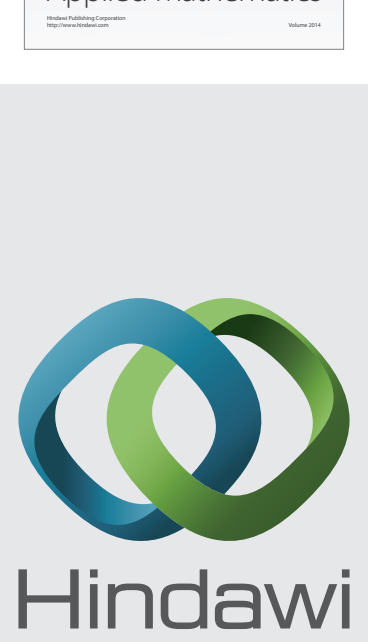

Submit your manuscripts at http://www.hindawi.com
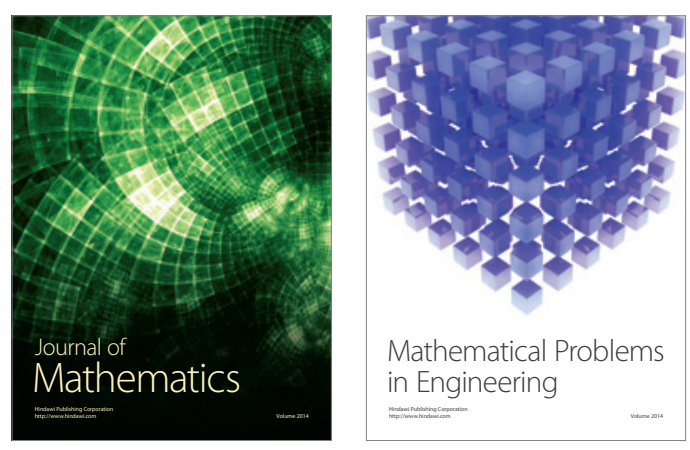

Mathematical Problems in Engineering
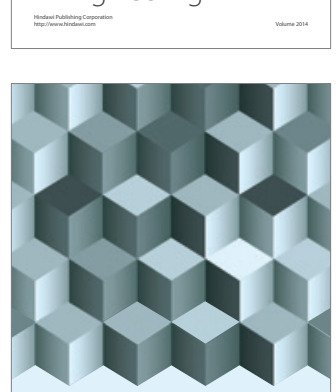

Journal of

Function Spaces
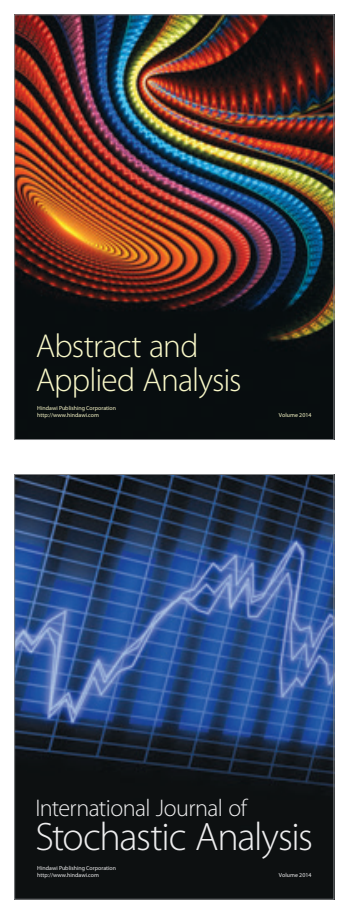

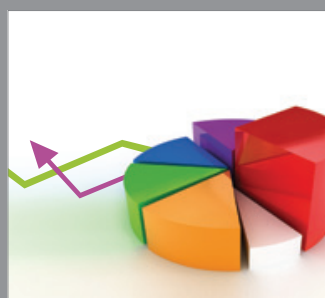

ournal of

Probability and Statistics

Promensencen
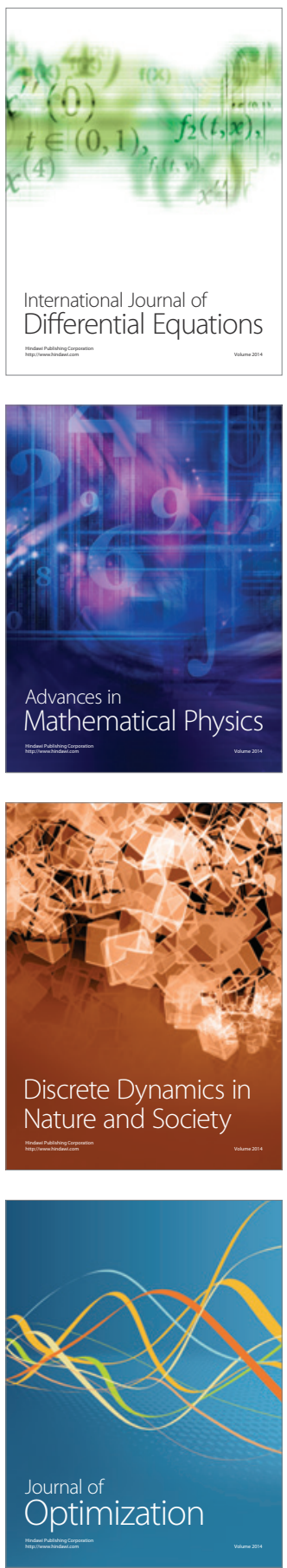